\title{
Behandlung der Hepatitis C gelingt meist ohne Interferon
}

Dank neuer "direct acting antivirals" (DAA) können heute über $90 \%$ der Patienten mit einer chronischen Hepatitis C ohne Interferongabe geheilt werden. Problematisch bleiben nur einige Risikogruppen.

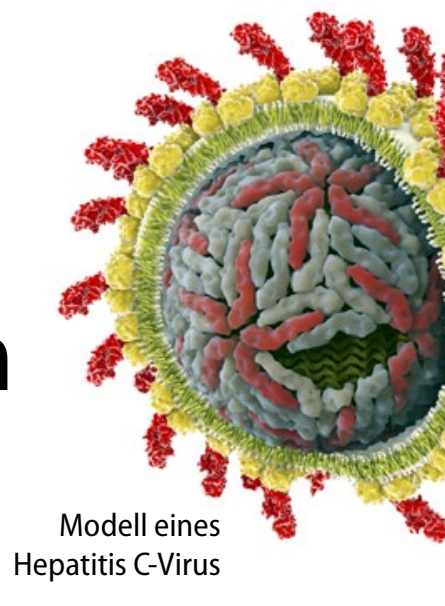

In Deutschland sind ca. 300.000 Menschen chronisch mit dem Hepatitis C-Virus (HCV) infiziert. Eine effektive antivirale Therapie dürfte dazu beitragen, die Inzidenz von HCV-assoziierter Leberzirrhose und hepatozellulärem Karzinom deutlich zu senken. Deshalb sollten alle Patienten mit erhöhten Leberwerten und Risikogruppen gescreent werden. Vorrangig behandelt werden sollten Patienten mit einer fortgeschrittenen Fibrose (F3-F4), Lebertransplantierte, Patienten mit extrahepatischen Manifestationen, solche mit einer HIV- oder HBV-Koinfektion oder anderen Lebererkrankungen, Typ-2-Diabetiker und Patienten mit Fatigue.

\section{Verschiedene Angriffspunkte}

Die DAAs greifen an verschiedenen Zielstrukturen im HCV-Lebenszyklus an. Dies sind die HCV-Protease, das HCVNS5-Protein und die HCV-Polymerase, wobei nukleotidische und nicht-nukleosidische Polymeraseinhibitoren unterschieden werden. Bei der Auswahl der Substanzen beim einzelnen Patienten

\begin{tabular}{l} 
Tab. 1 Antivirale Kombinationen je nach \\
HCV-Genotyp \\
Genotyp 1 \\
\hline Ledipasvir + Sofosbuvir \\
\hline Paritaprevir/Ritonavir+ Ombitasvir + Dasabuvir \\
\hline Simeprevir + Sofosbuvir \\
\hline Daclatasvir + Sofosbuvir \\
\hline Grazoprevir + Elbasvir \\
\hline Genotyp 2 \\
\hline Sofosbuvir + Ribavirin \\
\hline Genotyp 3 \\
\hline Sofosbuvir + Daclastavir \\
\hline Sofosbuvir + Ribavirin \\
\hline Ledispavir + Sofosbuvir + Ribavirin \\
\hline Genotyp 4 \\
\hline Ledipasvir + Sofosbuvir \\
\hline Paritaprevir/Ritonavir + Ombitasvir + Ribavirin
\end{tabular}

müssen Alter, viraler Genotyp (Tab. 1), Begleiterkrankungen, insbesondere eine gleichzeitig bestehende HBV- bzw. HIVInfektion, das Vorliegen einer Niereninsuffizienz, die Begleitmedikation, der Schweregrad der Lebererkrankung und der Vortherapiestatus ebenso berücksichtigt werden wie die antivirale Effektivität und die offiziellen Zulassungskriterien für die Substanz.

\section{Fortschritte auch bei \\ Problempatienten}

Doch es gibt Patientengruppen, bei denen die antivirale Therapie problematisch bleibt oder noch keine ausreichenden Daten vorliegen, um eindeutige Therapieempfehlungen geben zu können. Dazu gehören Patienten mit einer erfolglosen Vortherapie bzw. einer Resistenz, solche mit einer HIV- bzw. HBV-Begleitinfektion, einer chronischen Niereninsuffizienz, einer Leberzirrhose und/oder einer Infektion mit dem Genotyp 3.

Nach neueren Studienergebnissen zeigen bestimmte Kombinationen doch auch bei solchen Problempatienten eine vergleichbare Wirkung. Bei Patienten mit einer Leberzirrhose sollte die Therapie allerdings über 24 Wochen und in Kombination mit Ribavirin durchgeführt werden. Bei Patienten mit einer HIV-Begleitinfektion müssen Interaktionen mit den HIV-Medikamenten berücksichtigt werden.

Dr. Peter Stiefelhagen

\section{Kolorektale Neoplasie}

\section{NSAR verhindern weitere Darmpolypen}

In einer Studie zu fortgeschrittenen kolorektalen Neoplasien haben nichtsteroidale Antirheumatika vom Non-ASS-Typ erneute Neoplasien am ehesten verhindert.

US-Forscher haben 15 randomisierte und kontrollierte Studien zu zehn verschiedenen medikamentösen Strategien in der Sekundärprävention fortgeschrittener kolorektaler Neoplasien einer Netzwerk-Metaanalyse unterzogen. Untersucht wurden u.a. nichtsteroidale Antirheumatika (NSAR) vom Non-ASS-Typ, Acetylsalicylsäure (ASS), Kalzium, Vitamin D und Folsäure. Primärer Endpunkt der Vergleiche war das Auftreten fortgeschrittener metachroner Neoplasien innerhalb von drei bis fünf Jahren nach der Entfernung einer vorherigen kolorektalen Neubildung.

Das mediane Risiko für eine fortgeschrittene Neoplasie wurde durch NSAR vom Non-ASS-Typ um gut $60 \%$ reduziert. ASS in niedriger Dosis ( $\leq 160 \mathrm{mg} / \mathrm{Tag})$ senkte die Gefahr um rund $30 \%$, hoch dosiert ( $\geq 300 \mathrm{mg} / \mathrm{Tag}$ ) um $20 \%$. In diesen Größenordnungen bewegten sich auch die Kombinationen von ASS mit Folsäure bzw. mit Kalzium und Vitamin D.

Dr. Robert Bublak

Dulai PS et al. BMJ 2016; 355: i6188 